\title{
Merkwürdige Wege der HCV-Infektion im Krankenhaus
}

\author{
Wenn das Personal etwas Stoff für \\ sich abzweigt, bekommt der Patient \\ gelegentlich ein bleibendes An- \\ denken an seinen Krankenhausauf- \\ enthalt.
}

- In einem amerikanischen Krankenhaus fielen 2007/2008 drei Fälle von Hepatitis $\mathrm{C}$ bei Patienten ohne erkennbare Risikofaktoren auf. Molekulargenetische Untersuchungen ergaben, dass es sich dabei um denselben Virusstamm handelte. Die Akten der Patienten wurden daraufhin sorgfältig auf ein mögliches Risiko für eine iatrogene Hepatitis-CInfektion ausgewertet. Insbesondere achtete man darauf, zu welchem Zeitpunkt der Patient von welchem Mitglied des Personals ein Narkotikum verabreicht bekommen hatte.

Die Recherchen konzentrierten sich bald auf die Röntgen-Abteilung, wo alle drei Patienten im Rahmen einer interventionellen Untersuchung potenziell von einem der 21 dort beschäftigten radiologischen Assistenten Fentanyl bekommen hatten.

Nach intensiven Befragungen des gesamten Personals gab schließlich ein As- sistent zu, dass er seit 2004 immer wieder einmal Fentanyl zum Eigengebrauch abgezweigt hatte. Er verwendete dazu zwei verschiedene Methoden. Zum einen holte er Spritzen aus dem Abfallbehälter für scharfe Gegenstände und injizierte sich Fentanyl-Reste, die sich in einzelnen Spitzen befanden. Diese Technik bedeutete allerdings keine Gefährdung von Patienten. Manchmal verwendete er aber auch vorbereitete Spritzen, steckte eine feinere Kanüle auf, injizierte sich einige Milliliter selbst, ersetzte das Volumen durch Kochsalz und steckte die größere, ursprüngliche Nadel wieder auf die Spritze. Auf diese Weise musste es zur Kontamination von Spritzen gekommen sein, mit denen dann die Patienten infiziert wurden. In der Abteilung wurden auch noch Benzodiazepine und Hydromorphon verwendet. Von diesen Substanzen zweigte der Assistent aber nichts für sich ab.

Der Assistent war selbst mit HCV infiziert, wobei sein Virus eine hohe genetische Konkordanz mit den Viren der drei infizierten Patienten aufwies. Ein aufwändiges Screening-Programm an 4000 Patienten, die zwischen 2004 und
2010, das heißt bis zur Entlassung dieses Assistenten, in der Abteilung behandelt wurden, erbrachte zwei weitere Patienten, die auf diese Weise mit HCV infiziert worden waren.

\section{Kommentar}

Abgesehen von diesen vier Fällen wurden bislang in den USA nur vier weitere HCVÜbertragungen infolge Abzweigung von Narkotika durch Krankenhauspersonal berichtet. Eigentlich erstaunlich wenig, wenn man bedenkt, dass in einer Umfrage der amerikanischen Behörde für Drogenmissbrauch und psychische Gesundheit immerhin $4 \%$ einer repräsentativen Stichprobe von Krankenhauspersonal den Gebrauch illegaler Drogen zugab. In der Szene in Deutschland sind derzeit besonders gebrauchte Fentanyl-Pflaster in Altenheimen gefragt, die man aus dem Abfalleimer holt. Wenigstens werden dadurch keine Patienten gefährdet!

H. S. FüESSL -

- Hellinger WC et al.

(Korres.: Walter C. Hellinger, MD, Mayo Clicin, Division of Infectious Diseases, 4500 San Pablo Road, Jacksonville, FL 32224; helling@mayo. edu): Health-Care associated hepatitis $C$ infections attributed to narcotic diversion. Ann. Int Med. 2012; 156: 477-482

\section{Einseitig gealtert}

Ein 69-jähriger Mann stellte sich mit einer asymptomatischen Verdickung und Faltenbildung der Haut der linken Gesichtshälfte vor. Der Zustand hatte sich im Lauf von 25 Jahren ausgebildet. Bei der klinischen Untersuchung zeigten sich eine Hyperkeratose mit ausgeprägter Furchenbildung, multiple offene Komedonen und Hautareale mit nodulärer Elastose. In einer Hautbiopsie fanden sich eine Anhäufung von elastolytischem Material in der Dermis und die Ausbildung von Milia im Bereich der Follikel des Vellushaars. Diese Befunde sind vereinbar mit dem Favre-Racouchot-Syndrom, bekannt auch als Dermatoheliosis.
Der Patient gab an, dass er 28 Jahre lang in einem Fernlaster unterwegs war. UVA-Strahlen durchdringen Autoscheiben, penetrieren die Epidermis und die oberen Schichten der Dermis. Eine chronische UVA-Exposition führt zur Verdickung der Epidermis und des

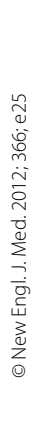

Stratum corneum sowie zur Zerstörung elastischer Fasern. UVA-Strahlen bewirken an der Haut hauptsächlich einen Alterungseffekt, verursachen aber auch DNA-Mutationen und toxische Effekte, die zur Ausbildung von Hautkrebs führen können. Der bedeutsamere Risikofaktor hierfür ist allerdings UVB-Strahlung. Dem Patienten wurde die regelmäßige Anwendung von Sonnenschutz, die laufende Inspektion zur Früherkennung von Hautkrebs und die Anwendung topischer Retinoide empfohlen. H. S. FüESSL .

- J.R.S. Gordon, J.C. Grieva

(Korres.: jrsgordon@gmail.com): Unilateral Dermatoheliosis. New Engl. J. Med. 2012; 366; e25 